\title{
Effect of Korean red ginseng on cold hypersensitivity in the hands and feet: study protocol for a randomized controlled trial
}

\author{
Kyoung-Sun Park', Jin-Woo Kim ${ }^{1}$, Jun-Young Jo ${ }^{1}$, Deok-Sang Hwang ${ }^{1}$, Chang-Hoon Lee ${ }^{1}$, Jun-Bock Jang ${ }^{1}$,
} Kyung-Sub Lee ${ }^{1}$, Inkwon Yeo ${ }^{2}$ and Jin-Moo Lee ${ }^{1 *}$

\begin{abstract}
Background: Cold hypersensitivity in the hands and feet (CHHF) is one of the most common complaints among Asians, especially in women. Korean red ginseng (KRG), which is a steamed form of Panax ginseng, has vasodilating action in the peripheral vessels and increases blood flow under cold stress. However, few studies have evaluated the effect of KRG on cold hypersensitivity.

Methods/Design: This trial is a randomized, double-blind, placebo-controlled trial in 80 CHHF patients. The trial will be implemented at Kyung Hee University Hospital at Gangdong in Seoul, Korea. The participants will take KRG or a placebo for eight weeks, after which they will be followed-up for four weeks. During the administration period, six capsules of $500 \mathrm{mg}$ KRG or placebo will be provided twice a day. The primary outcome is change of skin temperature in the hands between baseline and after treatment. The secondary outcomes include the visual analogue scale scores of cold hypersensitivity in the hands, change of skin temperature and the VAS scores of cold hypersensitivity in the feet, the recovery rate of the skin temperature by the cold stress test of the hands, the distal-dorsal difference of the hands, power variables of heart rate variability, and the 36-item short form health survey.
\end{abstract}

Discussion: This study is the first trial to evaluate the efficacy of KRG on CHHF by using infrared thermography. Our study will provide basic evidence regarding CHHF.

Trial registration: CliniacalTrials.gov NCT01664156

Keywords: Cold hypersensitivity in the hands and feet, Korean red ginseng, Infrared thermography, Cold stress test, Distal-dorsal difference

\section{Background}

Generally, cold hypersensitivity refers to the condition in which patients feel excessively cold at low temperatures, and cold hypersensitivity in the hands and feet (CHHF) is one of the most common complaints among Asians, especially in women. According to a previous study on the genetic etiology of CHHF, the ratio of CHHF among females to males was found to be approximately $3: 2$ [1]. In particular, the prevalence of CHHF in Japanese, Korean and Chinese women is $54.3 \%, 25 \%$ and $20 \%$, respectively [2]. CHHF can lower the quality of life by

\footnotetext{
* Correspondence: hanbang9597@hanmail.net

${ }^{1}$ Department of Gynecology, College of Korean Medicine, Kyung Hee University, Hoegi-dong, Dongdaemun-gu, Seoul 130-701, Republic of Korea Full list of author information is available at the end of the article
}

interfering with daily activities and, according to Korean medicine, may also be related to gynecological disorders such as menstrual pain, irregular bleeding, leucorrhea and infertility [3]. The known causes of CHHF are autonomic nervous dysfunction, peripheral neuropathy, median neuritis, anemia and diabetes mellitus [4]. However, the pathophysiology of CHHF remains unknown and a uniform treatment has not been established.

The root of Panax ginseng C.A. Meyer has been used worldwide for thousands of years for treatment of diverse diseases including diabetes [5], postmenopausal symptoms [6] and cancers [7]. Korean red ginseng (KRG) is a steamed form of Panax ginseng, which suggests that chemical transformation of active physiological properties occur involving ginsenosides, polysaccharides, peptides and polyacetylenic

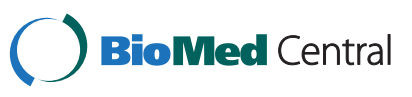

(c) 2013 Park et al.; licensee BioMed Central Ltd. This is an open access article distributed under the terms of the Creative Commons Attribution License (http://creativecommons.org/licenses/by/2.0), which permits unrestricted use, distribution, and reproduction in any medium, provided the original work is properly cited. 
alcohols [8]. As a consequence of these biochemical changes, KRG acquires additional physiological activities. Steaming ginseng at temperatures over $100^{\circ} \mathrm{C}$, results in the production of the gisenosides $\operatorname{Rg} 3$ and $\operatorname{Rg} 5$, which are not present in raw ginseng. Moreover, steamed ginseng is more potent at inducing endothelium-dependent vasodilation and radical-scavenging [9].

Many studies have suggested that KRG benefits vascular functions. KRG and its ginsenosides had vasodilating action on peripheral vessels and increased the blood flow under cold stress in animal experiments [10]. The ginsenosides isolated from KRG have been reported to cause vasodilation in an endothelium-dependent and nitric oxide- (NO-) mediated manner in a rabbit model [11]. Especially, the ginsenoside $\operatorname{Rg} 3$ was proven to inhibit vascular contraction as a consequence of $\mathrm{NO}$ production in a rat in vitro and in vivo [12]. Besides, in humans, KRG improved the vascular endothelial dysfunction in patients with hypertension through increasing synthesis of NO [13]. In addition, according to a randomized, controlled, double-blind trial, KRG was proven to improve arterial stiffness as measured by augmentation index [14]. According to Korean medicine, KRG is considered to have a warm quality and is used as a tonic to invigorate the body and enhance the ' $Q i$ ', which subsequently improves blood flow [15].

The vasodilating action and the warm quality of KRG provide a basis for its potential efficacy on CHHF. However, only one clinical trial has evaluated the effect of KRG on CHHF [16]. In the previous trial, the participants were divided into three groups that received KRG, placebo or nifedipine (a vasodilator). The participants then submerged their hands in cold water and the time until the pain became intolerable was measured. The tolerance time to cold stress significantly increased after administration of KRG compared to the placebo and nifedipine. However, the measurement of the tolerance time to cold stress used in the previous study is a subjective assessment tool.

The aim of the current study is to evaluate the efficacy of KRG on CHHF by using an objective assessment tool, infrared thermography. The hypothesis is that KRG will reduce $\mathrm{CHHF}$ more effectively than placebo after eight weeks of administering either KRG or placebo. The outcome measures include changes of skin temperature and the visual analogue scale (VAS) scores of CHHF, the recovery rate (RR) of skin temperature by the cold stress test (CST) of the hands, the distal-dorsal difference (DDD) of the hands, power variables of heart rate variability (HRV), and the 36-item short form health survey (SF-36).

\section{Methods/Design Trial design}

This trial is a randomized, double-blind, placebo-controlled trial in $80 \mathrm{CHHF}$ patients. The trial will be implemented at
Kyung Hee University Hospital at Gangdong in Seoul, Korea. The participants will take either KRG or placebo for eight weeks, after which they will be followed-up for four weeks. During the administration period, six capsules of $500 \mathrm{mg}$ KRG or placebo will be provided twice a day (one hour after breakfast and dinner). A flow chart of the entire trial is shown in Figure 1.

\section{Participants \\ Inclusion criteria}

The patients who meet all threeof the following inclusion criteria items will be included:

(1) Females aged 16 to 60 years

(2) Females complaining of CHHF

(3) A thermal difference greater than $0.3^{\circ} \mathrm{C}$ between the palm and the upper arm

\section{Exclusion criteria}

Patients who report the following conditions will be excluded:

(1)Skin ailments, radiculopathy, thrombophlebitis and injuries affecting infrared imaging

(2)Alcohol abuse or alcoholism

(3) A history of cancer within the past five years

(4) Severe depression or mental illness

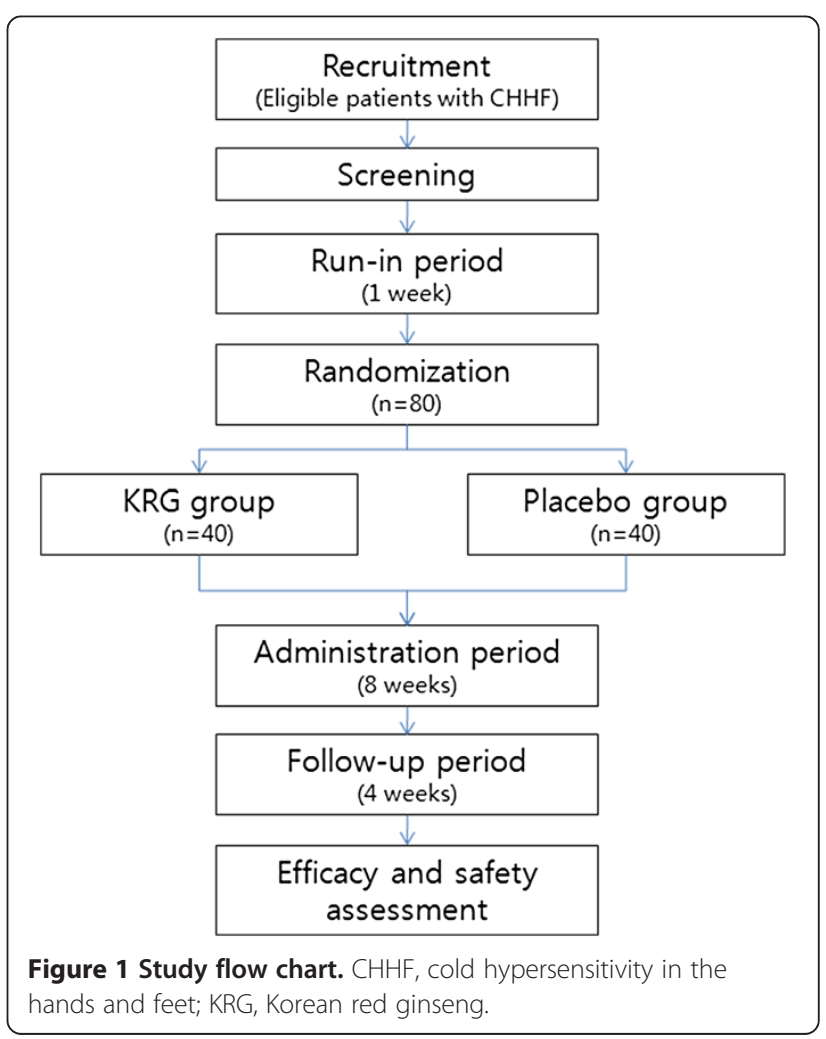


(5) Taking hypertensive or diabetic medications or thrombolytic agents

(6) Pregnant or breastfeeding

(7) Severe heart, pulmonary, hepatic and renal diseases (assessed by self-reporting and a blood test at the screening visit)

(8) Allergies to KRG or ginseng

(9) Ingestion of herbal medicine or nutritional supplements within a week before participation

(10) Participation in another clinical trial within the past three months

\section{Interventions}

The KRG (Korean Red Ginseng powder capsule ${ }^{\circledR}$ ) was manufactured by Korea Ginseng Corporation (Seoul, Korea) from the root of six-year-old Panax ginseng C.A. Meyer (family Araliaceae) harvested in Korea. The raw ginseng was cultivated and managed by Good Agricultural Practices established by the Rural Development Administration. The manufacture of KRG was processed according to Korean Good Manufacturing Practices and permitted and regulated by the Korean Food and Drug Administration. KRG was made by steaming fresh ginseng at 90 to $100^{\circ} \mathrm{C}$ for three hours, followed by drying at 50 to $80^{\circ} \mathrm{C}$. KRG capsules containing KRG powder were prepared from ground red ginseng (KRG capsule, $500 \mathrm{mg}$ /capsule). A total of $3 \mathrm{~g}$ of KRG powder contained $2 \mathrm{~g}$ of carbohydrates and $1 \mathrm{~g}$ of protein. The capsule was made of hydroxypropyl methylcellulose, pectin, purified water, sucrose fatty acid ester, glycerin, calcium gluconate and glacial acetic acid. KRG was analyzed by highperformance liquid chromatography. KRG contained the following active compounds: -Rb1, $5.61 \mathrm{mg} / \mathrm{g}$; -Rb2, 2.03 mg/g; -Rc, 2.20 mg/g; -Rd, 0.39 mg/g; -Re, $1.88 \mathrm{mg} / \mathrm{g}$; -Rf, $0.89 \mathrm{mg} / \mathrm{g} ;-\mathrm{Rg} 1,3.06 \mathrm{mg} / \mathrm{g} ;-\mathrm{Rg} 2(\mathrm{~s}), 0.15 \mathrm{mg} / \mathrm{g} ;-\mathrm{Rg} 3(\mathrm{r})$, $0.08 \mathrm{mg} / \mathrm{g} ;-\mathrm{Rg} 3(\mathrm{~s}), 0.17 \mathrm{mg} / \mathrm{g}$; -Rh1, $0.30 \mathrm{mg} / \mathrm{g}$, and other minor ginsenosides. The standard chemical components in KRG were the total amount of Rb1 and Rg1 (6 mg/g). The examination of the remaining agricultural chemical was also conducted. The tolerance limit for pesticide residue of KRG was $0.5 \mathrm{mg} / \mathrm{kg}$ according to the regulation of Korean Food and Drug Administration. The voucher specimens were kept at the laboratory of Korea Ginseng Corporation (Seoul, Korea). The lot number of KRG that will be used in our trial is 30032005.

The participants assigned to the KRG group will be required to take six capsules of $500 \mathrm{mg}$ KRG twice a day (one hour after breakfast and dinner), accounting for a total of $6 \mathrm{~g}$ in a day. The dosage of KRG was determined based on the total amount of Rb1 and Rg1, according to Korean Food Standards Codex. The participants assigned to the placebo group will take placebo capsules similar to the color, flavor and scent of KRG. Placebo was made of cornstarch, natural coloring (Brown
CG-11771, JEY's F.I. Inc., Sungnam Korea), brown caramel coloring (Bolak Co., Hwasong Korea) and red ginseng flavor (C80509, French Korean Aromatics Co., Yongin Korea). The lot number of placebo that will be used in our trial is 30032006. Samples of the placebo were also kept at the Korea Ginseng Corporation (Seoul, Korea). After several attempts, a placebo that could not be distinguished from the real KRG by 20 healthy persons was successfully produced. Besides, at the end of the study, all participants will be asked whether the experimental agents that they had taken were real or placebo in order to evaluate the success of blinding. In addition, the patients will be given a diary, which they will be asked to fill in twice a day, after taking KRG or placebo. The diary will be used to check their compliance to the administration. The patients' data will be excluded from the per-protocol analysis unless their compliance rate is more than $70 \%$.

\section{Objectives}

The objectives of the current study are (1) to evaluate the efficacy of KRG on CHHF and (2) to establish basic evidence via the evaluation of CHHF by using objective assessment tools.

\section{Outcomes}

\section{Primary outcome}

The primary outcome is a change of skin temperature in the hands between baseline (visit 2) and after treatment (visit 4). The skin temperature will be obtained by infrared thermography. For the infrared thermography examination, the patient will be asked to avoid hot showers, hot packs, smoking, exercise, acupuncture and stimulants such as caffeine for two hours before the examination. The patient will be acclimatized to room temperature $\left(25 \pm 1^{\circ} \mathrm{C}\right)$ for 15 minutes and seated comfortably on a chair without physiological or psychological stress. Subsequently, the patient will stand in an anatomical posture. Thermal images of the palm and the upper arm will be obtained to assess cold hypersensitivity in the hands. The difference between the palm and the upper arm, which is related to the severity of cold hypersensitivity in the hands, will then be calculated.

\section{Secondary outcome}

The secondary outcomes include the VAS scores of cold hypersensitivity in the hands, change of skin temperature and the VAS scores of cold hypersensitivity in the feet, RR of the skin temperature by the CST of the hands, the DDD of the hands, power variables of HRV, and the SF-36 between the baseline (visit 2) and after the treatment (visit 4). A 100-mm VAS measurement will be used to assess the severity of CHHF. The VAS scores will be rounded to the nearest integer in millimeters. 
The CST is used to examine the function of recovery after cold stress. The patient will be seated and the baseline thermal images of the dorsum of both hands will be acquired with infrared thermography. Subsequently, both hands will be submerged up to the wrist in cold water $\left(20^{\circ} \mathrm{C}\right)$ for a period of one minute. The hands will then be taken out of the cold water and will be carefully wiped and dried with a dry towel. Thermal images of the dorsum of both hands will be obtained immediately after immersion and six minutes later. The RR of the skin temperature at six minutes will be calculated as follows:

$$
\mathrm{RR}=\left(\mathrm{T}_{6}-\mathrm{T}_{0}\right) /\left(\mathrm{T}_{\text {base }}-\mathrm{T}_{0}\right) \times 100(\%)
$$

$\mathrm{T}_{\text {base: }}$ baseline skin temperature

$\mathrm{T}_{0}$ : skin temperature immediately after cold stress

$\mathrm{T}_{6}$ : skin temperature six minutes after cold stress

Recently, the thermographic DDD has been used as a parameter to evaluate the severity of vasoconstriction $[17,18]$. The DDD was calculated by subtracting the temperature of the finger from that of the dorsum [19]. Therefore, the DDD is considered positive if the finger is colder than the dorsum, and a high DDD indicates severe CHHF.

HRV is used to study the activity of the autonomic nervous system indirectly. Low-frequency (LF) power is thought to be strongly related to sympathetic nerve system activity and correlates with the regulation of blood pressure and peripheral vascular tone. High-frequency (HF) power reflects sinus arrhythmia and breathing activity and is strongly based on parasympathetic nerve system activity [20]. The ratio of LF to HF power is commonly interpreted as an indicator of the relative balance between sympathetic and parasympathetic activity, with higher ratios corresponding to higher relative sympathetic activity [21]. The total power is not a specific indicator of a particular type of autonomic nervous system expression, but is rather an indicator of the total amount of variability, which includes all of the different possible influences on heart rate variation [20].

CHHF may lower quality of life by interfering with daily life and restricting activities in the cold environment. Therefore, improving quality of life is another important goal in the treatment of CHHF. The SF-36 is a generic instrument to measure health-related quality of life and is widely used to survey physical and emotional health. The validated Korean version of SF-36 that will be used in this study was provided by the Health Assessment Laboratory (Boston, MA, USA). It consists of 36 questions grouped into eight dimensions: physical function (10 items), role limitations owing to physical health problems (4 items), bodily pain (2 items), general health perception (6 items), energy and vitality (4 items), social function (2 items), role limitations owing to emotional problems (3 items) and mental health (5 items). The number of questions for each health concept ranges from 2 to 10 , and the number of response options per question is either two (yes or no) or six (none, very mild, mild, moderate, severe or very severe). Each of the dimension scores are expressed as a value between 0 and 100, with higher scores representing better health status [22].

\section{Safety assessment}

All participants will be asked to record any adverse events in a diary during the administration and the follow-up period. All adverse events will be described in the case report form (CRF). The complete blood count $(\mathrm{CBC})$, erythrocyte sedimentation rate (ESR), liver function test and renal function test will be assessed to determine the safety of the treatments at the completion of KRG or placebo administration. These analyses will be performed at an accredited laboratory. All values detected in the laboratory will be recorded by the investigator in the CRF.

\section{Sample size}

This trial has a pilot characteristic to evaluate the efficacy of KRG on CHHF by using infrared thermography. Although there was no previous study that is the same in design, we calculated the sample size on the basis of a study that used similar variables. The previous study measured the tolerance time to cold stress to assess the efficacy of KRG on CHHF using a two-sided test, yielding a 5\% significance level [16]. The formula for estimating the sample size is as follows:

$$
n_{t}=n_{c}=\left\{\left(\mathrm{Z}_{\alpha / 2}+\mathrm{Z}_{\beta}\right)^{2} \sigma^{2}(\lambda+1) / \lambda\right\} /\left(\mu_{\mathrm{t}}-\mu_{\mathrm{c}}\right)^{2}
$$

In the previous study, the tolerance time to cold stress prolonged 0.35 minute in the KRG group compared with the control group $\left(\mu_{\mathrm{t}}-\mu_{\mathrm{c}}\right)$, and a mean standard deviation $(\sigma)$ was 0.84 . In our study, the ratio $(\lambda)$ of KRG to placebo is $1: 1$. With an $80 \%$ power $(1-\beta)$ and a $5 \%$ significance level $(\alpha)$, assuming $\mu_{\mathrm{t}}-\mu_{\mathrm{c}}=0.35$ and $\sigma=0.84$, a sample size of $n_{t}=n_{c}=32$ patients per group is needed $\left(n_{t}\right.$, number of patients in KRG group; $n_{c}$, number of patients in placebo group). Anticipating a drop-out rate of approximately $20 \%$, the total sample size should be more than 80 women.

\section{Randomization}

Randomization will be performed by an independent statistician by generating allocation numbers using a randomization allocation program. The participants will not be stratified. They will randomly be assigned into KRG and placebo groups at a 1:1 ratio. The investigator will be subsequently notified of the number assigned to 
each participant and the participants will be given a random number at their second visit. The allocation table of participants will be kept by an independent statistician until the end of the study.

\section{Blinding}

The patients will be blinded to the treatment that they are provided. The investigator and clinical pharmacist will be also blinded to the randomization. Only the independent statistician will be aware of the randomization.

\section{Ethical approval}

The protocol of this trial has been approved by the institutional review board (IRB) and the ethics committee of Kyung Hee University Hospital at Gangdong, Seoul, Korea. The permission number of this study is KHNMC$\mathrm{OH}-\mathrm{IRB} 2012-004$ and the protocol identification number on ClinicalTrials.gov is NCT01664156. Written informed consent will be obtained from all participants prior to enrollment, and patients will be given adequate time to declare if they wish to participate before signing the consent form.

\section{Recruitment}

Information will be sent by short message service (SMS) to patients with CHHF who were previously identified at the Kyung Hee University Hospital at Gangdong. Public advertisements will be placed in the newspaper and homepage of the hospital to recruit participants. Posters, brochures and banners will be placed inside the hospital.

\section{Concomitant therapy}

During the clinical trial, patients will be prohibited from taking any kind of drugs or therapies that might affect symptoms related to CHHF. These include herbal medicines, acupuncture, moxibustion, cupping and infrared treatment. Furthermore, the patients will be prohibited from taking hypertensive or diabetic medications and thrombolytic agents that affect vascular or endothelial functions. However, the patients will be permitted to take medications that would not affect CHHF. For example, medications for a common cold, stomachache, diarrhea and menstrual pain will be allowed.

\section{Statistical analysis}

An independent statistician will perform the statistical analyses of all data in a blinded manner. An efficacy analysis will be performed for both ITT (intention-to-treat) and PP (per-protocol) data set. Baseline characteristics such as age and body mass index (BMI) between the two groups will be compared using independent $t$-test or chi square test. The target variables for analysis in this study include the following: (1) change of skin temperature and VAS scores of cold hypersensitivity in the hands;
(2) change of skin temperature and VAS scores of cold hypersensitivity in the feet; (3) RR of the skin temperature by the CST of the hands; (4) the DDD of the hands; (5) power variables of HRV; and (6) the SF-36. All values will be presented as mean $\pm \mathrm{SD}$. The paired $t$-test will be used to compare data between the baseline (visit 2) and after the treatment (visit 4) in each group. Comparison between the two groups will be performed using independent $t$-test at each time. If there are significant differences between the two groups at the baseline, we will compare the variables using ANCOVA. All statistical analyses of data will be performed by using SPSS version 17.0 (SPSS Inc., Chicago, IL), with $P$ - values $<0.05$ representing statistical significance.

\section{Discussion}

CHHF cannot be dissociated from Raynaud's phenomenon (RP). A diagnosis of RP can be made when color changes occur in the skin of the digits of the hands and feet in response to cold temperatures or emotional stress [23]. RP is known to be caused by a transient cession of blood flow to the digits of the hands or feet [24] and is associated with various diseases, such as rheumatoid arthritis, scleroderma, dermatomyositis, hypertension and migraine [25]. However, RP can occur in the absence of these disorders, which is referred to as primary RP [26]. Although the pathophysiology of primary RP remains poorly understood, increases in sympathetic receptor activity in blood vessels, endothelial dysfunction and some central thermoregulatory defects have been suggested to contribute to the development of primary RP [24].

A survey was conducted regarding the prevalence of RP among 1,008 outpatients who complained of abnormal sensory symptoms in the hands and feet. The most common abnormal sensory symptoms, in order of descending frequency, were tingling, coldness and pain. Of the 510 patients (52\%) who answered "yes" to the question, "Are your extremities unusually sensitive to cold?", 290 patients (43\%) showed RP [27]. These results suggest that $\mathrm{CHHF}$, a conventional circulatory disorder, may be regarded as latent RP. Thus, it can be said that CHHF is RP without color changes of the hands and feet.

Infrared thermography, which has been used as a noninvasive investigative tool in medicine since the 1960s, can easily assess local differences in skin temperature. Infrared thermography is an easily applicable, wellestablished imaging method that can be used to create temperature maps of the skin with satisfying reproducibility $[28,29]$. Since cutaneous temperature depends on local blood perfusion and thermal tissue properties, infrared imaging provides important indirect information concerning circulation, thermal properties, and the thermoregulatory function of cutaneous tissues [30]. The subjective estimation of finger temperature has been shown to be 
closely related to finger temperature as measured by infrared thermography [31].

For the diagnosis of cold hypersensitivity in the hands, thermographic measurements will be performed at two paired areas (the palm and upper arm). A diagnostic cut-off value for cold hypersensitivity in the hands was determined in a previous study. When the criterion for the thermal difference between the palm and the upper arm was set as greater than $0.3^{\circ} \mathrm{C}$, the sensitivity and the specificity of the test were $94.0 \%$ and $90.0 \%$, respectively [4]. In the current study, we will diagnose CHHF according to this cut-off value.

The CST is applied to exposed skin areas with impaired thermoregulation [32]. The CST reportedly has high sensitivity and specificity for RP in comparison with healthy control subjects [33]. In addition, the CST was shown to have good sensitivity, specificity, and positive and negative predictive values for the diagnosis of digital vasospasm in patients with hand-arm vibration syndrome [34]. A previous study demonstrated a significant negative correlation between the RR of finger temperature after cold stress and VAS scores [35]. Therefore, RR after cold stress would be a good indicator for evaluating CHHF.

Among other methods, the measurement of skin temperature with the CST has been reported to be a good indicator of sympathetic responses [36], and has been used to evaluate central autonomic and peripheral vascular responses [37]. Cold stimulation increases sympathetic activity and the removal of the extremities from a cool environment results in prompt rewarming in the healthy subjects, with an RR of over $80 \%$ at five minutes. Therefore, a reduced RR indicates exaggerated sympathetic nerve function. According to previous works, a $R R$ of $<80 \%$ is considered clinically meaningful and suggests increased sympathetic nerve activities [38].

The sympathetic nervous system plays a major role in the regulation of cutaneous blood flow [39]. Therefore, sympathetic dysregulation has been hypothesized to be a factor in the genesis of RP. The exaggerated digital vasoconstrictor responses to cold, emotional stress, and postural changes [40] and the efficacy of both local anesthesia and sympathetic blockade in controlling vasoconstriction of RP [41] are consistent with a hypothesis of sympathetic over-activity. In a clinical examination, HRV spectrum analysis revealed significantly higher LF values in the RP group compared with the control group [42]. Another trial revealed relatively more sympathetic nerve system expression and less parasympathetic expression in a slow rewarming group after cold exposure compared to a normal group. Lower values were observed for all parameters after cold weather training for 15 months, and this was particularly evident for sympathetic nerve system expression, as indicated by lower LF values [43]. In the current study, we will evaluate autonomic nervous activity after the administration of KRG by using HRV.

Controversy exists in the literature regarding the effect of ginseng on autonomic nervous activity. Wild ginseng pharmacopuncture in healthy adults tended to activate the autonomic nervous system, particularly the sympathetic nervous system [44]. Another clinical trial showed higher parasympathetic and lower sympathetic activity modulation after the administration of Kampo including ginseng [45]. However, the investigators used wild ginseng pharmacopuncture or Kampo (a mixture of ginseng, oriental bezoar and glycyrrhiza), not ginseng alone. The current study is the first trial to evaluate autonomic nervous activity through the oral administration of KRG.

The DDD was shown to be a valuable parameter in discriminating between patients with primary RP and secondary RP due to systemic sclerosis. Previously, a DDD $>1^{\circ} \mathrm{C}$ in any finger at room temperature was considered a specific indicator of underlying structural vascular disease [19]. Because the DDD is used as a parameter to evaluate the severity of vasoconstriction, the severity of CHHF can be measured by the DDD.

Although CHHF is poorly defined medically, complaints of CHHF are common in Asian women. Nevertheless, few studies have been conducted regarding the diagnosis and treatment of CHHF. The current study is the first trial to evaluate the efficacy of KRG on CHHF by using infrared thermography. We anticipate that this trial will provide basic evidence regarding $\mathrm{CHHF}$ and that the protocol for this trial will be a reference for designing further clinical trials.

\section{Trial status}

The study was designed in 2012 and the first participant was randomized on 15 October 2012. Recruitment for this study is ongoing.

\section{Abbreviations \\ ANCOVA: Analysis of covariance; CBC: Complete blood count; CHHF: Cold hypersensitivity on hands and feet; CRF: Case report form; CST: Cold stress test; DDD: Distal-dorsal difference; ESR: Erythrocyte sedimentation rate; HRV: Heart rate variability; IRB: Institutional review boards; ITT: Intention-to-treat; KRG: Korean red ginseng; LF: Low frequency; PP: Per-protocol; RP: Raynaud's phenomenon; RR: Recovery rate; SD: Standard deviation; SF-36: The 36-item short form health survey; VAS: Visual analogue scale.}

\section{Competing interests}

The authors declare that they have no competing interests.

\section{Authors' contributions}

KSP, JWK, JYJ and JML contributed to the funding and the design of the study. SJH, NRY, DSH, CHL, JBJ and KSL participated in the design of the study. IKY calculated the sample size and determined the methods of statistical analysis. KSP and JML prepared the manuscript. All authors read and approved the manuscript.

\section{Acknowledgements}

This study was sponsored by the 2012 grant from the Korean Society of Ginseng funded by Korea Ginseng Corporation. 


\section{Author details}

${ }^{1}$ Department of Gynecology, College of Korean Medicine, Kyung Hee University, Hoegi-dong, Dongdaemun-gu, Seoul 130-701, Republic of Korea. 2Department of Statistics, College of Science, Sookmyung Women's University, Cheongpa 2 ga, Yongsan-gu, Seoul 140-742, Republic of Korea.

Received: 3 December 2012 Accepted: 4 December 2013

Published: 19 December 2013

\section{References}

1. Hur YM, Chae JH, Chung KW, Kim JJ, Jeong HU, Kim JW, Seo SY, Kim KS: Feeling of cold hands and feet is a highly heritable phenotype. Twin Res Hum Genet 2012, 15:166-169.

2. Lee $S L$, Lee $K S$, Song BK: The literature review on the cold hypersensitivity of women. J Korean Gynecol 1996, 9:55-80.

3. Bae GM, Cho HS, Kim KK, Lee IS: The research on the cold hypersensitivity of patients in the gynecology clinic. J Korean Gynecol 2002, 15:101-113.

4. Kim DH, Kim YS, Lee KS: Standardization of diagnosis of cold hypersensitivity of hands and feet by D.I.T.I. J Korean Gynecol 2001 14:129-134

5. Vuksan V, Sung MK, Sievenpiper UL, Stavro PM, Jenkins AL, Di Buono M, Lee KS, Leiter LA, Nam KY, Arnason JT: Korean red ginseng (Panax ginseng) improves glucose and insulin regulation in well-controlled, type 2 diabetes: results of a randomized, double-blind, placebo-controlled study of efficacy and safety. Nutr Metab Cardiovasc Dis 2008, 18:46-56.

6. Kim SY, Seo SK, Choi YM, Jeon YE, Lim KJ, Cho S, Choi YS, Lee BS: Effects of red ginseng supplementation on menopausal symptoms and cardiovascular risk factors in postmenopausal women: a double-blind randomized controlled trial. Menopause 2012, 19:461.

7. Helms S: Cancer prevention and therapeutics: Panax ginseng. Altern Med Rev 2004, 9:259-274

8. Park JD: Recent studies on the chemical constituents of Korean ginseng (Panaxginseng CA Meyer). Korean J Ginseng Sci 1996, 20:389-415.

9. Kim WY, Kim JM, Han SB, Lee SK, Kim ND, Park MK, Kim CK, Park JH: Steaming of ginseng at high temperature enhances biological activity. J Nat Prod 2000, 63:1702-1704.

10. Kaneko H, Nakanishi K: Vasodilator action of ginsenosides. Clin Study 1984 61:2657-2662

11. Chen X: Cardiovascular protection by ginsenosides and their nitric oxide releasing action. Clin Exp Pharmacol Physiol 1996, 23:728-732.

12. Kim ND, Kim EM, Kang KW, Cho MK, Choi SY, Kim SG: Ginsenoside Rg3 inhibits phenylephrine-induced vascular contraction through induction of nitric oxide synthase. Br J Pharmacol 2003, 140:661-670.

13. Sung J, Han KH, Zo JH, Park HJ, Kim CH, Oh BH: Effects of red ginseng upon vascular endothelial function in patients with essential hypertension. Am J Chin Med 2000, 28:205-216.

14. Jovanovski E, Jenkins A, Dias AG, Peeva V, Sievenpiper J, Arnason JT, Rahelic D, Josse RG, Vuksan V: Effects of Korean red ginseng (Panax ginseng C. A. Mayer) and its isolated ginsenosides and polysaccharides on arterial stiffness in healthy individuals. Am J Hypertens 2010, 23:469-472.

15. Kwan C: The effects of different ginseng extracts on vascular contraction in vitro: evidence for Yin-Yang principle. Acta Phytother 1999, 2:73-77.

16. Kaneko $H$, Nakanishi $K$ : Proof of the mysterious efficacy of ginseng: basic and clinical trials: clinical effects of medical ginseng, Korean red ginseng specifically, its anti-stress action for prevention of disease. J Pharmacol Sci 2004, 95:158-162.

17. Clark S, Hollis S, Campbell F, Moore T, Jayson M, Herrick A: The distal-dorsal difference as a possible predictor of secondary Raynaud's phenomenon. J Rheumatol 1999, 26:1125-1128.

18. Pauling JD, Flower V, Shipley J, Harris ND, McHugh NJ: Influence of the cold challenge on the discriminatory capacity of the digital distal-dorsal difference in the thermographic assessment of Raynaud's phenomenon. Microvasc Res 2011, 82:364-368.

19. Anderson M, Moore T, Lunt M, Herrick A: The 'distal-dorsal difference': a thermographic parameter by which to differentiate between primary and secondary Raynaud's phenomenon. Rheumatology 2007, 46:533-538.

20. Montano N, Ruscone TG, Porta A, Lombardi F, Pagani M, Malliani A: Power spectrum analysis of heart rate variability to assess the changes in sympathovagal balance during graded orthostatic tilt. Circulation 1994 90:1826-1831.
21. Perini $R$, Veicsteinas $A$ : Heart rate variability and autonomic activity at rest and during exercise in various physiological conditions. Eur J Appl Physiol 2003, 90:317-325.

22. Ware JE Jr, Sherbourne CD: The MOS 36-item short-form health survey (SF-36): I. Conceptual framework and item selection. Med Care 1992, 30:473-483.

23. Levien TL: Advances in the treatment of Raynaud's phenomenon. Vasc Health Risk Manag 2010, 6:167.

24. Cooke JP, Marshall JM: Mechanisms of Raynaud's disease. Vasc Med 2005 10:293-307

25. Gayraud M: Raynaud's phenomenon. Joint Bone Spine 2007, 74:e1-e8.

26. Wigley FM: Raynaud's phenomenon. N Engl J Med 2002, 347:1001-1008.

27. Choi WS, Ock SM, Byeon JH, Jung SH, Park KS, Park JW, Yoo DJ, Lee JB, Kim CM: The prevalence of diagnosis as Raynaud's disease among the people complaint of abnormal sensation on hands and feet. Korean J Fam Med 2003, 24:1085-1091.

28. Zaproudina N, Varmavuo V, Airaksinen O, Närhi M: Reproducibility of infrared thermography measurements in healthy individuals. Physiol Meas 2008, 29:515.

29. Murray AK, Moore TL, Manning JB, Taylor C, Griffiths CEM, Herrick AL: Noninvasive imaging techniques in the assessment of scleroderma spectrum disorders. Arthritis Rheum 2009, 61:1103-1111.

30. Di Carlo A: Thermography and the possibilities for its applications in clinical and experimental dermatology. Clin Dermatol 1995 13:329.

31. Polunina A, Gugleta K, Kochkorov A, Katamay R, Flammer J, Orgül S: Relationship between peripheral blood flow in extremities and choroidal circulation. Klin Monatsbl Augenheilkd 2011, 228:302-305.

32. O'Reilly D, Taylor L, El-Hadidy K, Jayson M: Measurement of cold challenge responses in primary Raynaud's phenomenon and Raynaud's phenomenon associated with systemic sclerosis. Ann Rheum Dis 1992, 51:1193-1196.

33. Schuhfried O, Vacariu G, Lang T, Korpan M, Kiener HP, Fialka-Moser V: Thermographic parameters in the diagnosis of secondary Raynaud's phenomenon. Arch Physical Med Rehabil 2000, 81:495-499.

34. Coughlin P, Chetter I, Kent P, Kester R: The analysis of sensitivity, specificity, positive predictive value and negative predictive value of cold provocation thermography in the objective diagnosis of the hand-arm vibration syndrome. Occup Med 2001, 51:75-80.

35. Han JY, Joe JH, Jang JB, Kim YS, Lee KS: Cold stress test for the diagnosis of cold hypersensitivity on hands. J Oriental Med Thermol 2003, 2:17-23.

36. Kistler A, Mariauzouls C, von Berlepsch K: Fingertip temperature as an indicator for sympathetic responses. Int J Psychophysiol 1998, 29:35-41.

37. Faes TJ, Wagemans MF, Cillekens JM, Scheffer GJ, Karemaker JM, Bertelsmann FW: The validity and reproducibility of the skin vasomotor test-studies in normal subjects, after spinal anaesthesia, and in diabetes mellitus. Clin Auton Res 1993, 3:319-324.

38. Zaproudina N, Lipponen JA, Eskelinen P, Tarvainen MP, Karjalainen PA Närhi M: Measurements of skin temperature responses to cold exposure of foot and face in healthy individuals: variability and influencing factors. Clin Physiol Funct Imaging 2011, 31:307-314.

39. Abramson Dl: Neural regulation of cutaneous circulation. Adv Biol Skin 1972, 12:207.

40. Olsen N, Petring OU, Rossing N: Exaggerated postural vasoconstrictor reflex in Raynaud's phenomenon. Br Med J (Clin Res Ed) 1987, 294:1186-1188.

41. Nielsen S, Christensen N, Olsen N, Lassen N: Raynaud's phenomenon: peripheral catecholamine concentration and effect of sympathectomy. Acta Chir Scand Suppl 1980, 502:57

42. Koszewicz M, Gosk-Bierska I, Bilinska M, Podemski R, Budrewicz S, Adamiec R, Slotwinski K: Autonomic dysfunction in primary Raynaud's phenomenon. Int Angio/ 2009, 28:127-131.

43. Brändström H, Wiklund U, Karlsson M, Ängquist KA, Grip H, Haney M: Autonomic nerve system responses for normal and slow rewarmers after hand cold provocation: effects of long-term cold climate training. Int Arch Occup Environ Health 2013, 86:357-365.

44. Yook TH, Yu JS, Lee HY, Song BY, Kim LH, Roh JD, Shin JC, Lim ST: Comparing the effects of distilled Rehmanniaglutinosa, wild ginseng and astragali radix pharmacopuncture with Heart Rate Variability (HRV): a 
randomized, sham-controlled and double-blind clinical trial. J Acupunct Meridian Stud 2009, 2:239-247.

45. Zheng A, Moritani T: Effect of the combination of ginseng, oriental bezoar and glycyrrhiza on autonomic nervous activity and immune system under mental arithmetic stress. J Nutr Sci Vitaminol 2008, 54:244-249.

doi:10.1186/1745-6215-14-438

Cite this article as: Park et al.: Effect of Korean red ginseng on cold hypersensitivity in the hands and feet: study protocol for a randomized controlled trial. Trials 2013 14:438.

\section{Submit your next manuscript to BioMed Central and take full advantage of:}

- Convenient online submission

- Thorough peer review

- No space constraints or color figure charges

- Immediate publication on acceptance

- Inclusion in PubMed, CAS, Scopus and Google Scholar

- Research which is freely available for redistribution 